\title{
Desarrollo de instrumentos virtuales en Excel como apoyo a la enseñanza de la metrología
}

\section{Development of virtual instruments in Excel to support the teaching of metrology}

Presentación: 28/08/2019

Aprobación: 26/08/2020

\begin{abstract}
Juan Marcos Banegas
Centro de Investigación y Desarrollo en Ingeniería Eléctrica y Sistemas Energéticos CIESE - Facultad Regional Santa Fe - Universidad Tecnológica Nacional - Argentina

jmbanegas@frsf.utn.edu.ar
\end{abstract}

\section{Matías Orué}

Centro de Investigación y Desarrollo en Ingeniería Eléctrica y Sistemas Energéticos CIESE - Facultad Regional Santa Fe - Universidad Tecnológica Nacional - Argentina

morue@frsf.utn.edu.ar

\section{Edgardo Cámara}

Centro de Investigación y Desarrollo en Ingeniería Eléctrica y Sistemas Energéticos CIESE - Facultad Regional Santa Fe - Universidad Tecnológica Nacional - Argentina

ecamara@frsf.utn.edu.ar

\section{Resumen}

La tecnología como medio para la mejora del proceso de enseñanza-aprendizaje, ya sea a través del contacto físico en el aula o laboratorio, o por medio de sistemas virtuales en línea, tiene una potencialidad altamente positiva, haciendo posible profundizar la capacidad de asimilación de conceptos dando una nueva dimensión al aprendizaje significativo. En este trabajo, se exploran diferentes tecnologías útiles para la capacitación en metrología, y se realizan propuestas para el desarrollo de diferentes instrumentos virtuales en planillas Excel, como un recurso de fácil acceso por su masiva distribución en los ambientes educativos. Esta herramienta demostró su versatilidad tanto para el profesor como para el alumno, y su portabilidad permite una fácil migración entre diferentes plataformas con un simple intercambio de archivos. Cabe aclarar que el software utilizado para el desarrollo de los instrumentos virtuales ocupa un lugar privilegiado en la práctica metrológica dentro de los laboratorios de calibración, haciéndolo extremadamente competitivo, incluso frente a sus competidores gratuitos. 
Palabras claves: Laboratorio virtual, laboratorio remoto, Excel, metrología, calibración, instrumentación, instrumento virtual.

\begin{abstract}
Technology as a means to improve the teaching-learning process, whether through physical contact in the classroom or laboratory, or through online virtual systems, has a highly positive potential, making it possible to deepen the assimilation capacity of concepts giving a new dimension to meaningful learning. In this work, explore different technologies useful for training in metrology, and develop proposals for the development of different virtual instruments in Excel spreadsheets, as an easily accessible resource due to its massive distribution in educational environments. This tool demonstrates its versatility for both the teacher and the student, and its portability allows easy migration between different platforms with a simple file exchange. It should be clarified that the software used for the development of virtual instruments occupies a privileged place in metrological practice within calibration laboratories, making it extremely competitive, even with its free competitors.
\end{abstract}

Keywords: Virtual laboratory, remote laboratory, Excel, metrology, calibration, instrumentation, virtual instrument.

\title{
1. Introducción
}

El aprendizaje significativo es impensable sin la experimentación práctica, más aún cuando nos referimos a áreas duras del conocimiento relacionados a la técnica y la tecnología, donde el experimento de laboratorio es crucial. Con estas actividades no solo se persigue la formación práctica de los estudiantes como una característica aislada, sino que permiten conceptualizar la impronta en el medio real de la teoría impartida en el aula, además desarrollar confianza en dicho conocimiento y desarrollar un espíritu curioso y creativo a través de nuevas preguntas ("qué pasaría si ..."). Sin embargo, hay varios problemas con los que lidiar, como: limitaciones de tiempo y espacio, tiempos muertos de preparación de equipos y sistemas auxiliares, limitaciones de cantidad de equipamiento versus alumnos con la consecuente pérdida de atención de algunos estudiantes al trabajar en grupos, entre otros (Odema et al., 2019: 214-218).

Por otra parte, no es novedad que la tecnología está transformando la educación: proporcionando una variedad de herramientas para la enseñanza / aprendizaje, que van desde simples ayudas para la enseñanza en el aula hasta educación en línea, sin embargo, se han identificado muchos problemas relacionados con la implementación efectiva de las tecnologías modernas respecto del aprendizaje a distancia de nivel universitario; sin embargo, el aprendizaje a distancia a través de los cursos tipo e-learning se está convirtiendo en el modo de aprendizaje más relevante y más demandado en la última década en la educación superior (Leontyeva, 2018). En este contexto, el "aprendizaje combinado" (Blended Learning - combina la interacción personal de las sesiones de clase tradicionales, con tecnologías en línea), demostró ser más efectivo para mejorar el aprendizaje de los estudiantes (Bender et al., 2006: 114-122). Inclusive, es muy notorio que los alumnos de los últimos años, no aprecian completamente las lecciones tradicionales en el aula, lo que lleva a la pérdida de motivación e interés (Ballu et al., 2016: 148-153).

En este sentido, el uso de la tecnología para complementar la educación tradicional 
parece ser una buena opción, especialmente para complementar la capacitación práctica. De hecho, a lo largo de los años se desarrollaron muchas herramientas tecnológicas diferentes, lo que les permite a los estudiantes realizar experimentos desde una computadora, sin la necesidad de estar físicamente presentes en los laboratorios: estos se denominan laboratorios en línea.

En este documento, se resumen y analizan diferentes tecnologías de educación en línea con respecto a su aplicabilidad para la enseñanza de la metrología. Luego, se plantean objetivos específicos, a fin de seleccionar una herramienta adecuada para enseñar conceptos de metrología. Considerando estos objetivos, se propone y desarrolla una nueva herramienta de simulación de instrumentos virtuales, especialmente diseñada para la enseñanza de metrología, utilizando una hoja de cálculo de Excel.

\subsection{Laboratorios en línea}

Básicamente, hay dos tipos principales de laboratorios en línea: laboratorios remotos y virtuales. Los laboratorios remotos (RL por sus siglas en inglés) utilizan hardware real para realizar experimentos reales. Se puede acceder al control y la instrumentación a través de Internet, y en la mayoría de los casos se puede monitorear en video en tiempo real. Por otro lado, los laboratorios virtuales (VL por sus siglas en inglés) usan software de simulación (es decir, no hay equipos reales involucrados). Algunos de ellos incluso cuentan con un entorno 3D para una experiencia más realista (Ballu et al., 2016: 148-153).

\subsubsection{Laboratorios remotos (RL).}

La matriz más común para RL es el sistema de dos niveles: el laboratorio y el usuario. En el primer caso es básicamente un laboratorio real con equipos y hardware reales. Los controles para la operación física del equipo, los sensores para toma de medidas se conectan a un servidor informático por medio de interfaces electrónicas y transductores, al que se puede acceder en línea. Por el lado del usuario es la computadora del alumno que se usa para visualizar y controlar el laboratorio remoto por medio de alguna interfaz gráfica (Alli et al., 2015).

Se pueden encontrar muchos ejemplos de RL en la bibliografía que cubre una amplia variedad de aplicaciones, la mayoría de ellas relacionadas con entornos industriales o electrónicos. Se utiliza un software robusto conocido para la interfaz humano-máquina (HMI), como LabView (Alli et al., 2015) o sistemas SCADA (Z. Aydogmus et al., 2009: 126-132).

Muchas veces, se utiliza tecnologías de hardware reconfigurable basado en FPGA (siglas en inglés para matriz de compuertas lógicas programable en campo), lo que permite una mayor flexibilidad y versatilidad (K. Efstathiou et al., 2007: 1-6).

La principal ventaja de los RL es que se están realizando experimentos reales con equipos reales, pero sin la necesidad de estar físicamente presente en el laboratorio, eliminando las limitaciones de tiempo y espacio. Por otro lado, los laboratorios remotos tienen otros problemas relacionados con los costos (equipos y software propietario), acceso limitado de clientes remotos, problemas organizativos relacionados con el uso del laboratorio por parte de diferentes usuarios (R. Langmann et al., 2016: 130-135).

\subsubsection{Laboratorios virtuales (VL).}

La mayoría de estos problemas se pueden resolver utilizando herramientas multimedia para simular experimentos. Hay dos posibilidades principales con respecto a VL: una es usar sistemas de desarrollo integrado comercialmente disponibles (LabView, Measurement 
Studio, entre otros); otra opción es desarrollar una aplicación personalizada, generalmente utilizando programación orientada a objetos (por ejemplo: applets de Java) (D. Grimaldi et al., 2009: 485-493). Cada alternativa tiene puntos fuertes y débiles: costos, operabilidad, especificidad, capacidades de programación, consumo de tiempo y versatilidad, entre otros.

Las VL se presentan como una muy buena opción para complementar la educación y la capacitación en metrología, mediante el uso de instrumentos virtuales. Algunos ejemplos de tales aplicaciones incluyen: applets de Java para simular instrumentos de metrología dimensional como calibradores y micrómetros (A. Ballu et al., 2016: 148-153) (F. Al-Zahrani, 2010), simulación LabView de instrumentación virtual en el campo de las vibraciones (G. R. Gillich et al., 2010: 3806-3810) y herramientas desarrolladas específicamente como VIEW (Virtual Instrumentation Workbench for Instrumentation) para instrumentación electrónica como generador de señales, analizador de espectro y osciloscopio (J. M. G. Palop et al., 2000: 15-18). Algunos VL incluso tienen entornos 3D realistas y simulaciones complejas que cubren una variedad de experimentos (B. F. Woodfield et al., 2009). En el aula se comprueba fácilmente que tales herramientas pueden ser muy positivas para los estudiantes (J. M. Molina Jordá, 2013: 3355-3365).

Un uso adicional de las NTICs, que ha tomado mucho protagonismo en los últimos años, es el uso de herramientas interactivas para la enseñanza (S. Machado et al., 2006) (M. Farkhadov et al., 2018: 206-212). Presentan entornos multimedia interactivos para guiar al estudiante a través del proceso de aprendizaje mediante diferentes lecciones y actividades. La mayoría de estos sistemas de enseñanza no son realmente VL, ya que no hay posibilidad de realizar experimentos.

\subsubsection{RLs vs VLs para la enseñanza de metrología.}

Las VL presentan varias ventajas sobre las RL con respecto a su aplicación a la enseñanza de la metrología. En primer lugar, dado que no se necesita equipo real, los costos se reducen y no se necesita espacio. Además, los instrumentos virtuales suelen ser altamente personalizables para adaptarse a las necesidades de enseñanza, lo que permite sistemas más versátiles. De hecho, hay muchas más aplicaciones de VL en metrología, en lugar de RL.

El uso de la instrumentación virtual para enseñar temas específicos implica encontrar una aplicación comercial lista para usar que se adapte a sus necesidades o desarrollar las suyas propias. Incluso con el uso de entornos de desarrollo como LabView, se necesita conocimiento de programación. Y, en cualquier caso, la portabilidad está limitada debido a la necesidad de tener el software instalado en la computadora del estudiante.

\section{Motivación y objetivos.}

El objetivo principal de este trabajo fue desarrollar una herramienta simple y portátil para la simulación de instrumentos virtuales que se aplicará en la enseñanza de metrología, basada en los siguientes aspectos:

- Simplicidad: la herramienta debe ser simple, tanto para el profesor en la etapa de desarrollo (no se requieren habilidades de programación ni conocimientos específicos de software), como para los estudiantes en la práctica. Debería ser posible personalizar, modificar o agregar nuevas características de una manera muy simple.

- Portabilidad: la herramienta debe usarse en diferentes computadoras, e incluso en la nube, con requisitos mínimos de software, con una simple transferencia de archivos. No debe ser necesaria la instalación de software o licencias. 
- Especificidad: la herramienta está diseñada para simular instrumentos virtuales para enseñar conceptos relacionados con la metrología (en particular, aplicados a la metrología eléctrica) (JCGM 200, 2012).

Por lo tanto, debe ser capaz de simular diferentes tipos de instrumentos (tanto digitales como analógicos), incluyendo errores sistemáticos y aleatorios.

En este trabajo, se utilizó una hoja de cálculo de Microsoft Excel para simular diferentes tipos de instrumentos, considerando que los aspectos mencionados podrían abordarse adecuadamente con el uso de ese software. Aunque Excel no es un programa gratuito para el público en general, Office 365 Education tiene licencia gratuita para estudiantes y educadores, y la mayoría de las universidades les permite a los estudiantes usar una licencia de estudiante especial para Microsoft Office (A. Nebesová et al., 2017: 56-62). Otra ventaja relacionada con el uso de Excel es su amplia aplicación en los laboratorios de metrología (J. M. Banegas et al., 2016: 733), lo que la convierte en una herramienta perfecta para la capacitación de técnicos en metrología.

\section{Desarrollo de instrumentos virtuales en Excel.}

En este capítulo se describen los tipos de instrumentos que se desarrollaron, y sus potencialidades a la hora de simular situaciones reales de la práctica en metrología; dejando para siguientes capítulos diferentes actividades llevadas a cabo en el aula que demuestran la potencialidad y simplicidad mendionadas entre los objetivos.

\subsection{Interfaz gráfica para instrumentos digitales.}

El diseño de la interfaz gráfica de usuario se considera una parte muy relevante del proyecto; los detalles conducen a un entorno gráfico realista y motiva al estudiante a usar la herramienta, desarrollar la actividad en un ambiente conocido y aprender jugando sin riesgos de daño.

Para esta implementación, se utilizaron un calibrador FLUKE 5522A y las imágenes del multímetro digital FLUKE 115. Para simular las pantallas LCD, se utiliza la fuente de tipo verdadero "DJB Get Digital" (esta fuente está disponible en línea y es gratuita para uso no comercial). Usando cualquier software de edición de mapa de bits, la pantalla del instrumento se pinta en un color liso; luego, utilizando la función "seleccionar color transparente" en las herramientas de formato de imagen, las pantallas se vuelven transparentes y permiten ver el valor de la celda detrás de la imagen del instrumento. 


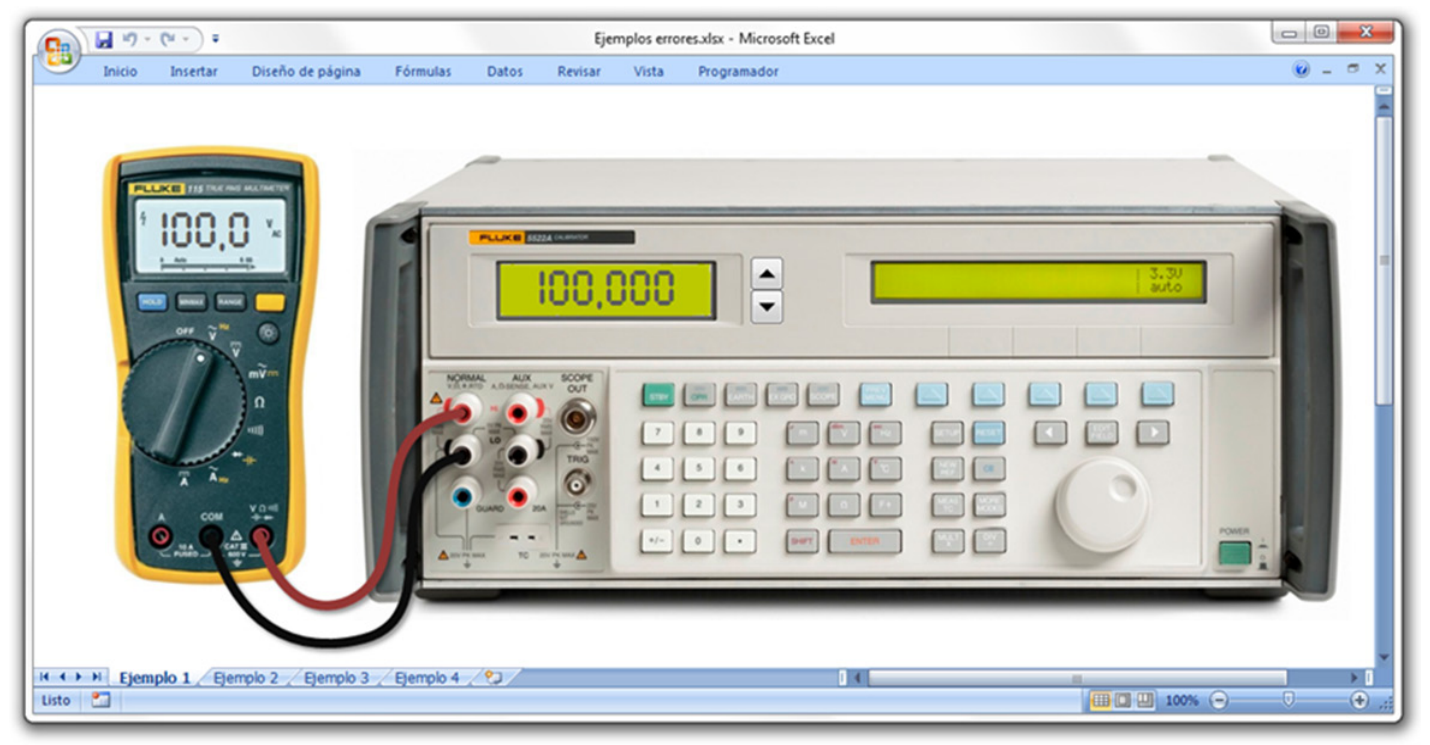

Fig. 1. Diseño de la interfaz gráfica de usuario para instrumentos digitales.

La figura 1 muestra el resultado final. Se utilizan dos celdas para los datos: una corresponde al valor de referencia generado por el calibrador ( $\mathrm{Vr})$ y la otra al valor medido en el dispositivo bajo prueba $(\mathrm{Vm})$. Una función tan simple como $\mathrm{Vm}=\mathrm{Vr}$ puede usarse para vincular ambos valores.

Para mejorar el realismo, se puede usar una tabla para seleccionar automáticamente la resolución para el valor que se muestra en el multímetro, simulando la función de rango automático del multímetro. La Fig. 2 muestra una tabla de ejemplo para un multímetro típico de 311/2 dígitos, con rangos de 1,999 - 19,99 - 199,9 - $1000 \mathrm{~V}$ (observe la forma correcta de introducir los valores y las resoluciones en la tabla). Con esa tabla, la siguiente función proporcionará el valor medido con la resolución adecuada (donde Vm es el valor medido, celda H3 en este caso): = TEXTO (Vm; VLOOKUP (Vm; C2: D6; 2)).

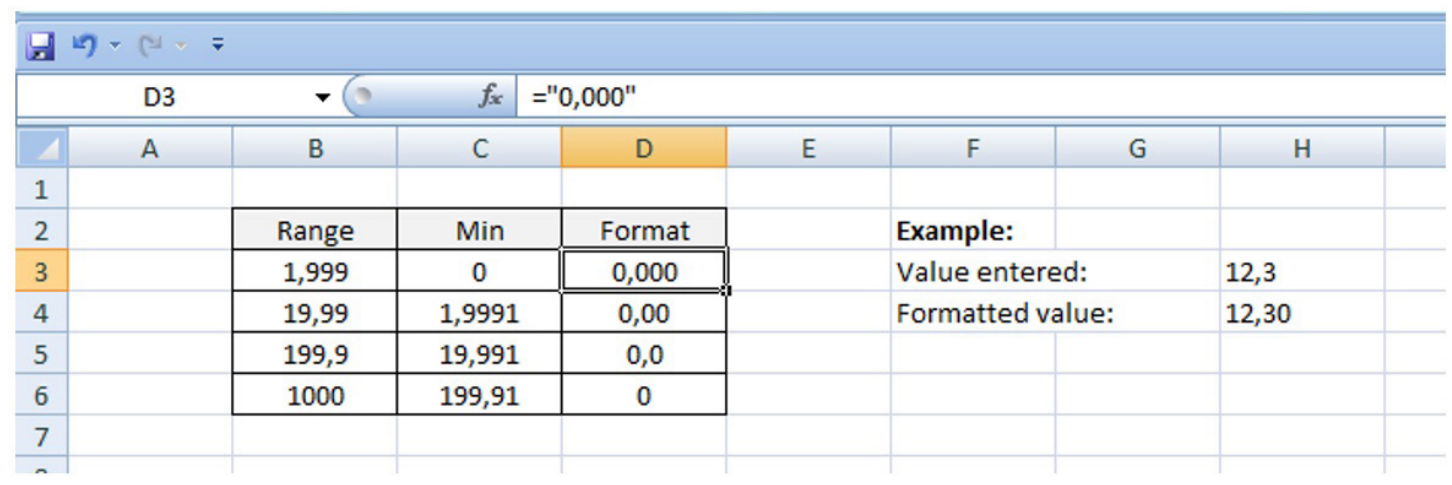

Fig. 2. Tabla de datos para simular diferentes rangos y resoluciones. 


\subsection{Interfaz gráfica para instrumentos analógicos.}

Desarrollar una interfaz de instrumento analógica requiere un poco más de trabajo. La base del diseño es una imagen del instrumento a simular (en este caso, un voltímetro analógico de $300 \mathrm{~V})$.

El puntero se simula con una línea en un gráfico XY. Los ejes se configuran de -1 a +1 , por lo que el centro del gráfico se utiliza como el pivote del puntero. La línea se dibuja usando dos puntos: uno es siempre $(0,0)$ - el pivote - y el otro se calcula con una fórmula simple para apuntar la línea en la dirección deseada - la punta - dependiendo del valor medido.

El gráfico se configura con un fondo transparente, los ejes se ocultan y la imagen se coloca junto al gráfico, con el pivote correspondiente al punto $(0,0)$ del gráfico. En este caso, se agregó una pequeña forma libre delante del gráfico para ocultar la parte pivotante de la línea y hacer que se vea más realista, sumado a un efecto de sombra que se aplica a la línea.

La Fig. 3 muestra el resultado final para el voltímetro analógico. Como en el primer caso, una celda se usa para ingresar el valor de referencia (Vr) y la otra celda para el valor medido (Vm). Esta celda está oculta a los estudiantes, por lo que solo pueden leer el valor medido de la escala del instrumento.
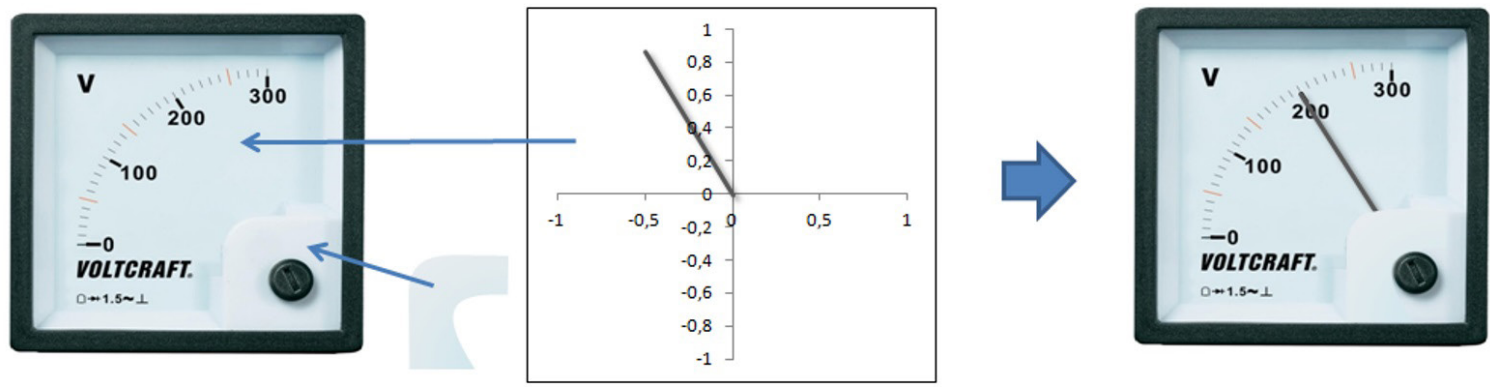

Fig. 3. Interfaz gráfica para instrumentos analógicos que muestra sus partes

\subsection{Simulando diferentes tipos de errores.}

En un instrumento ideal, el valor medido debe ser exactamente el valor de referencia $(\mathrm{Vm}=\mathrm{Vr})$. Sin embargo, los instrumentos reales (incluidos los instrumentos estándar, como el calibrador) tienen errores que se pueden simular en Excel. Se pueden simular diferentes errores y luego agregarlos al valor de referencia Vr y/o al valor medido Vm. Para facilitar el uso de la hoja de cálculo, es conveniente que cada tipo de error se calcule en una celda diferente y finalmente se agregue por completo a la referencia y/o al valor medido.

\subsubsection{Bias.}

También llamado error de cero, representa un error constante en toda la escala. Simplemente se suma al valor medido; por ejemplo, deje que ErrZ sea la magnitud del sesgo, luego: $V m=V r+E r r Z$.

\subsubsection{Error de span.}

Representa un error relativo constante en toda la escala, lo que significa que el error absoluto aumenta (o disminuye) junto con el valor medido. Deje que ErrS sea la magnitud 
del error de rango expresado como relativo al valor medido, luego para error de ganancia positiva:

$$
V m=V r \cdot(1+E r r S)
$$

\subsubsection{Error de precision.}

La falta de repetibilidad del instrumento se puede simular con la función aleatoria en Excel. Deje que ErrP sea el error de máxima precisión absoluta, luego:

$$
V m=V r+R A N D(-E r r P ; \operatorname{Err} P)
$$

Este valor cambia cada vez que el usuario ingresa un valor en cualquier celda.

\subsubsection{Error de linealidad.}

Para simular respuestas no lineales del instrumento, se puede usar un polinomio. Una forma muy simple es elegir algunos valores de error a lo largo de la escala y usar la curva de ajuste polinómica para obtener los coeficientes. Por ejemplo, el siguiente gráfico muestra un instrumento con errores de 0,05 en el 40\% de la escala y 0,1 en el 100\% de la escala:

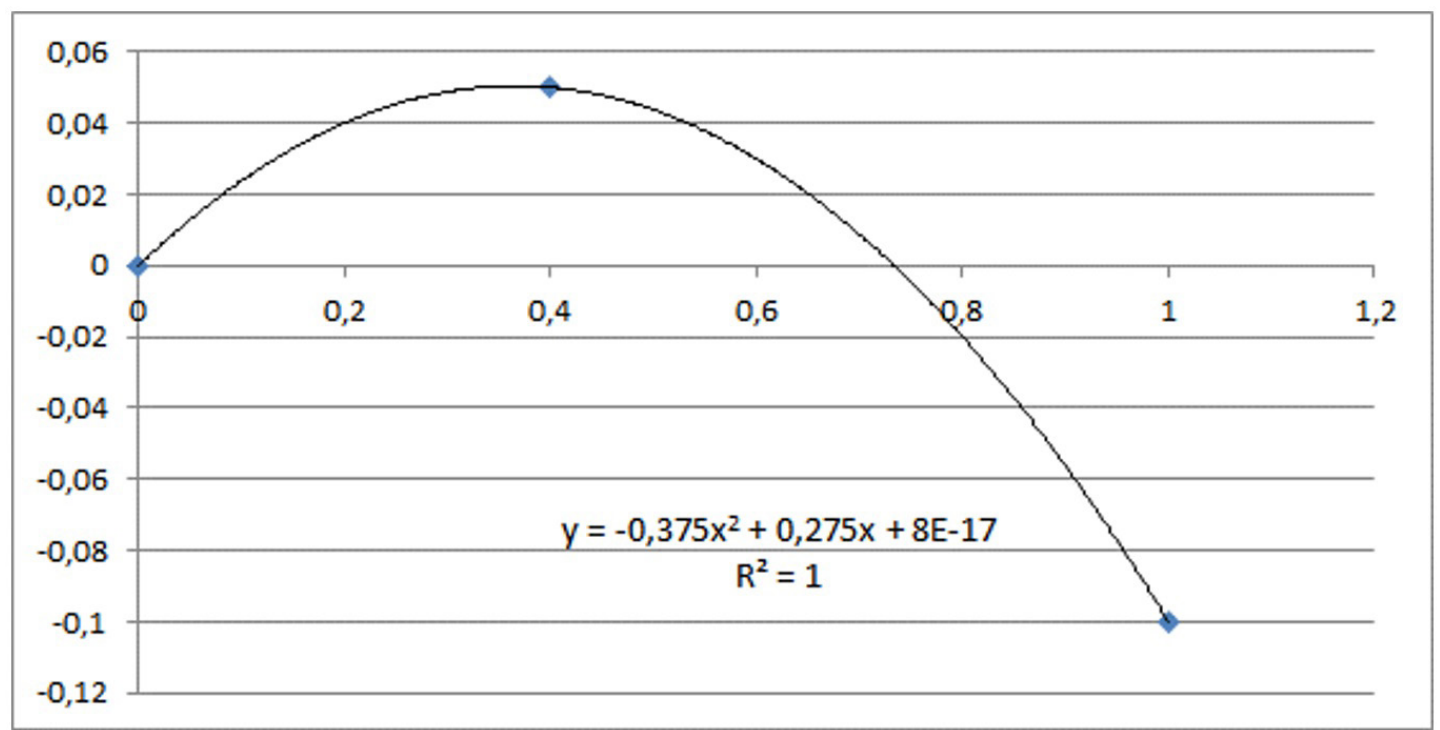

Fig. 4. Simulación de respuesta no lineal del instrumento.

Usando los coeficientes de ajuste, el valor medido da como resultado:

$$
V m=-0,375 * V r^{2}+0,275 * V r
$$

Nota: el último término se puede ignorar, ya que no necesitamos un ajuste exacto en este caso.

Otra opción es utilizar polinomios conocidos para simular instrumentos o sensores específicos, como termómetros de resistencia de platino (PRT) (J.M. Banegas et al., 2013), 
termopares y otros, según la aplicación.

Simulación de ajustes de intervalo cero.

Algunos instrumentos, como los controladores de temperatura y otros controladores industriales, cuentan con ajustes de cero e intervalo. Esto se puede simular fácilmente en Excel, y es muy útil para enseñar cómo configurar dichos dispositivos para minimizar los errores de medición.

La Fig. 5 muestra un controlador de temperatura virtual. Además de la celda Vm, se usan dos celdas más para ingresar los coeficientes cero y de span. Por lo general, en este instrumento, el valor cero se agrega al valor medido, mientras que el valor de span se multiplica por el valor medido; entonces:

$$
V m=\operatorname{span} \cdot V m{ }^{\prime}+\text { cero }
$$

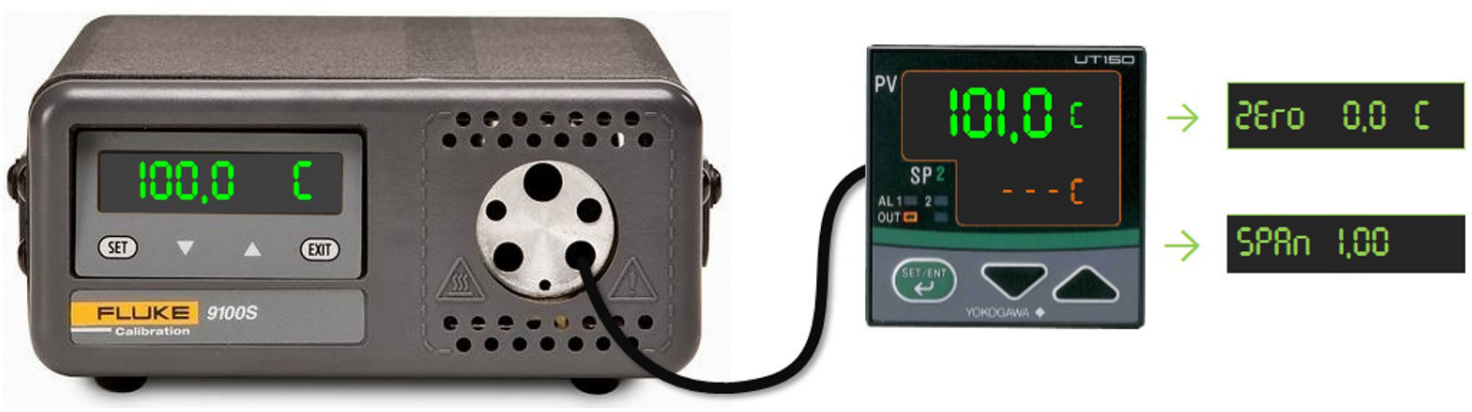

Fig. 5. Simulación de ajustes de cero y span en un controlador de temperatura

\section{Actividades propuestas utilizando instrumentos virtuales.}

Una vez que los instrumentos están listos, se pueden desarrollar varias actividades para enseñar diferentes conceptos sobre metrología. Aquí hay unos ejemplos. Estas actividades se utilizan para enseñar metrología en diferentes cursos en la Universidad Tecnológica Nacional, Facultad Regional Santa Fe. Los instrumentos virtuales utilizados en este trabajo están disponibles de forma gratuita previa solicitud al autor correspondiente.

\subsection{Calibración}

Usando la configuración que se muestra en la Fig. 1, es posible realizar una calibración virtual de un instrumento digital. Con esta actividad, se pueden abordar varios conceptos importantes de metrología (precisión, exactitud, veracidad, errores sistemáticos y aleatorios, correcciones).

Primero, un libro de Excel está organizado con 2 o más hojas, cada una con una configuración como se muestra en la Fig. 1. Cada multímetro virtual está configurado con una combinación diferente de errores, como se expresa en 2.3. La Fig. 6 muestra ejemplos de 2 respuestas multimétricas configuradas para esta actividad. 

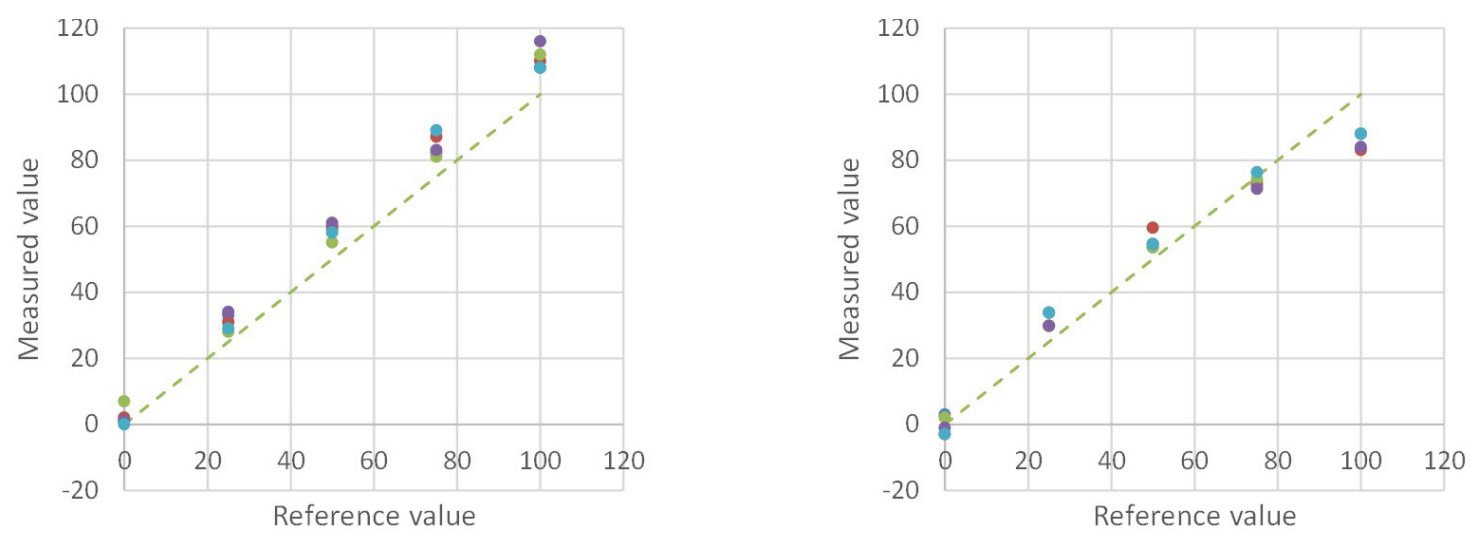

Fig. 6. Dos ejemplos de respuesta multimétrica: izquierda: cero + intervalo + errores de repetibilidad; derecha: linealidad + errores de repetibilidad.

Luego, se les pide a los estudiantes que realicen lo que necesiten para identificar los diferentes errores de los instrumentos y determinar su magnitud (se supone que deben realizar una calibración, repitiendo cada punto varias veces para estimar la repetibilidad). También se les pide que determinen las correcciones que deben aplicarse a cada instrumento para compensar los errores sistemáticos y verificar sus conclusiones simulando otros valores y aplicando las correcciones. Dado que los errores aleatorios todavía están presentes, el concepto de incertidumbre también se puede abordar en esta actividad.

Cuando el personal de capacitación de un laboratorio tiene un sistema de calidad (ISO/ IEC 17025, 2017), parte de la capacitación puede consistir en hacer un certificado de calibración, incluida la estimación de incertidumbre.

\subsection{Ajuste}

Usando la configuración que se muestra en la Fig. 5, es posible capacitar a los estudiantes para que realicen los ajustes de cero y de ganancia en controladores industriales. Previamente, el instrumento se configura con un error de cero (ver 2.3.1.) y de span (ver 2.3.2.) conocidos. Para dificultad adicional, también se puede configurar un pequeño error de repetibilidad.

En esta actividad, se les pide a los estudiantes que encuentren el mejor conjunto de coeficientes de ganancia y rango, los apliquen al instrumento virtual y verifiquen sus resultados con simulaciones adicionales. Por supuesto, el primer paso es realizar una calibración como se indica en 3.1.

\subsection{Comparaciones interlaboratorios}

Esta actividad es útil para abordar conceptos como la compatibilidad metrológica, Z-score, comparación interlaboratorios y otros conceptos estadísticos, y de aseguramiento de la validez de resultados de laboratorios (de ensayos o calibraciones). En este caso, varios archivos diferentes de Excel serán preparados; el dispositivo bajo prueba debe ser el mismo en todos los casos (este será asumido como el patrón viajero), pero el instrumento utilizado como patrón para cada estudiante o grupo (por ejemplo: el calibrador) puede variar. Inclusive, para propósitos didácticos, algunos de los instrumentos patrón están configurados intencionalmente con errores más grandes. 
Luego se pide a los estudiantes que realicen una calibración en puntos específicos e informen sus resultados, incluida una estimación de la incertidumbre de la medición. Finalmente, todos los resultados se compilan y procesan como en una prueba de competencia real, y los resultados se analizan junto a los demás participantes del curso.

\section{Resultados}

Los instrumentos virtuales aquí mostrados se vienen desarrollando desde el año 2018, a partir de la necesidad manifiesta de diferentes cátedras relacionadas con la medición de magnitudes eléctricas, temperatura, presión, etc., en las carreras de Ingeniería Eléctrica y Mecánica, como en las tecnicaturas de la Facultad Regional Santa Fe, además de cursos de capacitación sobre incertidumbres de medición, medidas en laboratorios, entre otros. Dicha necesidad surge de la poca disponibilidad de equipamiento para desarrollo de trabajos prácticos de laboratorio, donde se requiere mayor cantidad de instrumental para que el total de alumnos interactúe correcta y cabalmente, y en un entorno lo más cercano posible a la realidad, con las ventajas de poder reproducir las prácticas fuera del espacio físico del laboratorio.

Hasta el momento solo se tienen resultados cualitativos del impacto producido por el uso de esta herramienta en los grupos de alumnos que tuvieron contacto, observándose como característica principal, la rápida adaptación de los mismos al entorno, replicando fácilmente la lógica de manipulación de los instrumentos explicada en por el docente y sin el reparo típico por el "temor a dañar".

Por otra parte, se puede observar la correcta conceptualización de una medición de magnitudes físicas asociada a las características de una señal o su valor (tensión, temperatura, etc) representadas en display, y las cualidades de un instrumento particular (resolución, clase, incertidumbres, etc).

Se espera seguir con el desarrollo de nuevos instrumentos durante los próximos años, pero más precisamente se prevé desarrollar indicadores para obtener datos sobre la asimilación de estos conocimientos por parte de los alumnos.

\section{Conclusiones}

El uso de instrumentos virtuales para la enseñanza de conceptos de metrología a los estudiantes es muy útil, reforzando su asimilación al incorporar entornos físicos simulados. Los estudiantes son motivados al realizar este tipo de actividades, ya que pueden hacer experiencias virtuales sin el riesgo de dañar equipos realmente costosos. Además, la posibilidad de personalizar escenarios específicos ayuda al profesor a mostrar conceptos particulares (por ejemplo, diferentes tipos de errores).

En particular, Excel tiene muchas ventajas sobre otros sistemas:

- Es muy fácil configurar diferentes tipos de instrumentos y personalizar su comportamiento de acuerdo con las necesidades didácticas.

- No se necesita programación, todo se hace con la ayuda de algunas funciones simples incluidas en el paquete básico del software. Además, se pueden conseguir sistemas con una buena apariencia visual.

- Si se requiere un comportamiento más complejo, se puede desarrollar macros utilizando Visual Basic para aplicaciones. En este caso, aunque es más complejo, las posibilidades son prácticamente ilimitadas. 
- Es muy sencillo de distribuir y usar, ya que solo se necesita una transferencia de archivos. Se puede utilizar incluso en un teléfono inteligente y con aplicaciones en línea, por lo que no se requieren licencias costosas. Hace que estos instrumentos virtuales sean especialmente útiles para cursos en línea o cuando hay un campus virtual disponible. También se puede verificar su potencialidad al momento del repaso de conceptos por parte del estudiante en forma individual.

Por supuesto, la experiencia real en un laboratorio no puede ser reemplazada por completo con el uso de instrumentos virtuales, pero esto resulta en una excelente complementación para los estudiantes, ya que pueden realizar tantos experimentos como lo necesiten, sin limitarse en el tiempo o la ubicación. 


\section{Referencias}

M. Odema, I. Adly and H. A. Ghali (2019). "LabVIEW-Based Interactive Remote Experimentation Implementation using NI myRIO," 2019 International Conference on Innovative Trends in Computer Engineering, ITCE, Aswan, Egypt, February 2 to 4, 214-218.

I. A. Leontyeva (2018). "Modern Distance Learning Technologies in Higher Education: Introduction Problems", EURASIA Journal of Mathematics, Science and Technology Education, 14 (10), em1578.

D. M. Bender and J. D. Vredevoogd (2006). "Using online education technologies to support studio instruction”, Journal of Educational Technology \& Society, 9 (4), 114-122.

A. Ballu, X. Yan, A. Blanchard, T. Clet, S. Mouton and H. Niandou (2016). "Virtual Metrology Laboratory for e-Learning”, Procedia CIRP, 43, 148-153.

K. Alli, C.I. Ogboi, D. Ale, A.O. Ajibade and F. Oladipo (2015). "A LabView Based Online Control Experiments for Students' Learning”, Proceedings of the World Congress on Engineering and Computer Science, WCECS 2015, San Francisco, USA, October 21 to 23, 308-313.

Z. Aydogmus, O. Aydogmus (2009). "A web-based remote access laboratory using SCADA", IEEE Trans. Educ., 52 (1), 126-132.

K. Efstathiou, D. Karadimas and K. Zafeiropoulos (2007). "A Remote Electrical Engineering Laboratory based on Re-Configurable Hardware", 2007 IEEE Instrumentation \& Measurement Technology Conference, IMTC 2007, Warsaw, Poland, 1-6.

R. Langmann, H. Jacques (2016), "Generic remote labs in automation engineering", IEEE Glob. Eng. Educ. Conf., EDUCON, 10, April 13, 130-135.

D. Grimaldi and S. Rapuano (2009), "Hardware and software to design virtual laboratory for education in instrumentation and measurement”, Measurement, 42 (4), 485-493.

F. Al-Zahrani (2010), "Web-Based Learning and Training for Virtual Metrology Lab." JOURNAL OF TELECOMMUNICATIONS, 1 (2), 44-54.

G. R. Gillich, D. Frunzaverde, N. Gillich and D. Amariei (2010). "The use of virtual instruments in engineering education”, Procedia - Social and Behavioral Sciences, 2 (2), 3806-3810.

J. M. G. Palop and J. M. A. Teruel (2000). "Virtual work bench for electronic instrumentation teaching", IEEE Transactions on Education, 43 (1), 15-18.

B. F. Woodfield, M. C. Asplund and S. Haderlie (2009). "Virtual ChemLab, General Chemistry Laboratories v.2.5. Student Component”. Pearson, Prentice Hall, Nueva Jersey, United States.

J. M. Molina Jordá (2013). "Virtual Tools: Virtual Laboratories for Experimental science An Experience with VCL Tool”, Procedia - Social and Behavioral Sciences, 106, 3355-3365.

S. Machado and M. A. Cavaco (2006). "An interactive tool for teaching metrology", XVIII IMEKO WORLD CONGRESS - Metrology for a Sustainable Development, September 17 to 22, Rio de Janeiro, Brazil.

M. Farkhadov, A. Eliseev and M. Farkhadova (2018). "Remote Teaching System Based on 
Modern Multimedia Interactive Computer Technologies," 14th International Conference on Electronics Computer and Computation (ICECCO), Kaskelen, Kazakhstan, 206-212.

BIPM, IEC, IFCC, ILAC, ISO, IUPAC, IUPAP and OIML (2012). "JCGM 200 - International vocabulary of metrology - Basic and general concepts and associated terms (VIM)", 3rd edition.

< https://www.bipm.org/utils/common/documents/jcgm/JCGM_200_2012.pdf>

Microsoft Corporation (2019). “Office 365”.

< https://www.microsoft.com/en-us/education/products/office/default.aspx>

A. Nebesová and J. Sekničková (2017). "Graphical solution of linear programming problems in MS excel”, Turkish Online Journal of Educational Technology, (Special Issue INTE), November 1, 56-62.

J. M. Banegas and M. W. Orué (2016). "Software validation applied to spreadsheets used in laboratories working under ISO/IEC 17025”. Journal of Physics: Conference Series. 733 (1).

< https://iopscience.iop.org/article/10.1088/1742-6596/733/1/012071>

J. M. Banegas, J. Acosta, R. Furlani (2013). "Desarrollo de polinomios para hallar la inversa de la ecuación de Callendar - Van Dusen”. 10 Congreso Internacional de Metrología Eléctrica (X SEMETRO), Buenos Aires, Argentina.

SO/IEC 17025 (2017). "General requirements for the competence of testing and calibration laboratories", 3rd edition. 